\title{
Efficacy of Danlou Tablet in Patients with Non-ST Elevation Acute Coronary Syndrome Undergoing Percutaneous Coronary Intervention: Results from a Multicentre, Placebo-Controlled, Randomized Trial
}

\author{
Lei Wang, ${ }^{1,2}$ Xujie Zhao, ${ }^{1,2}$ Shuai Mao, ${ }^{1,2}$ Shaonan Liu, ${ }^{3}$ Xinfeng Guo, ${ }^{3}$ \\ Liheng Guo, ${ }^{1,2}$ Tinghai Du, ${ }^{4}$ Haiyu Yang, ${ }^{5}$ Fuhai Zhao, ${ }^{6}$ Keng Wu, ${ }^{7}$ Hongliang Cong, \\ Yang Wu, ${ }^{9}$ Phillip C. Yang, ${ }^{10}$ Keji Chen, ${ }^{6}$ and Minzhou Zhang ${ }^{1,2}$ \\ ${ }^{1}$ Department of Critical Care Medicine, 2nd Affiliated Hospital of Guangzhou University of Chinese Medicine, \\ Guangzhou 510120, China \\ ${ }^{2}$ Chest Pain Center, Guangdong Provincial Hospital of Chinese Medicine, Guangzhou 510120, China \\ ${ }^{3}$ China-Australia International Research Centre for Chinese Medicine (CAIRC-CM), Guangdong Provincial Academy of \\ Traditional Chinese Medicine, Guangzhou 510120, China \\ ${ }^{4}$ Department of Cardiovascular Medicine, First Affiliated Hospital of Henan College of TCM, Zhengzhou 450004, China \\ ${ }^{5}$ Department of Cardiovascular Medicine, Wuyi Hospital of TCM of Jiangmen City, Jiangmen 529000, China \\ ${ }^{6}$ Department of Cardiology, Xiyuan Hospital, Chinese Academy of Traditional Chinese Medicine, Beijing 100091, China \\ ${ }^{7}$ Department of Cardiology, Affiliated Hospital of Guangdong Medical College, Zhanjiang 524023, China \\ ${ }^{8}$ Department of Cardiology, Tianjin Chest Hospital, Tianjin 300051, China \\ ${ }^{9}$ Department of Cardiovascular Medicine, Oriental Hospital, Beijing University of Traditional Chinese Medicine, \\ Beijing 100078, China \\ ${ }^{10}$ Department of Cardiovascular Medicine, Stanford University School of Medicine, Stanford, CA 94305, USA
}

Correspondence should be addressed to Minzhou Zhang; minzhouzhang@aliyun.com

Received 15 June 2016; Revised 10 September 2016; Accepted 5 October 2016

Academic Editor: Chang-Gue Son

Copyright (c) 2016 Lei Wang et al. This is an open access article distributed under the Creative Commons Attribution License, which permits unrestricted use, distribution, and reproduction in any medium, provided the original work is properly cited.

\begin{abstract}
This study seeks to investigate potential cardioprotection of Danlou Tablets in patients undergoing PCI with non-ST elevation acute coronary syndrome (NSTE-ACS). 219 patients with NSTE-ACS were randomised to Danlou Tablet pretreatment $(n=109)$ or placebo $(n=110)$. No patients received statins prior to PCI and all patients were given atorvastatin $(10 \mathrm{mg} /$ day $)$ after procedure. The main endpoint was the composite incidence of major adverse cardiac events (MACEs) within 30 days after PCI. The proportion of patients with elevated levels of cTn I $>5 \times 99 \%$ of upper reference limit was significantly lower in the Danlou Tablet group at $8 \mathrm{~h}$ $(22.0 \%$ versus $34.5 \%, p=0.04)$ and $24 \mathrm{~h}(23.9 \%$ versus $38.2 \%, p=0.02)$ after PCI. The 30 -day MACEs occurred in $22.0 \%$ of the Danlou Tablet group and $33.6 \%$ in the placebo group $(p=0.06)$. The incidence of MACE at 90 -day follow-up was significantly decreased in the Danlou Tablet group compared to the placebo group $(23.9 \%$ versus $37.3 \%, p=0.03)$. The difference between the groups at 90 days was the incidence of nonfatal myocardial infarction ( $22 \%$ versus $34.5 \%, p=0.04)$. These findings might support that treatment with Danlou Tablet could reduce the incidence of periprocedural myocardial infarction in patients with ACS undergoing PCI.
\end{abstract}

\section{Introduction}

Over the past several decades, percutaneous coronary intervention (PCI) has emerged as the predominant therapeutic administration for ischemic heart disease. However, the high incidence of periprocedural myocardial infarction (PMI) following PCI severely impaired the benefits of coronary revascularization. It has been demonstrated that approximately 


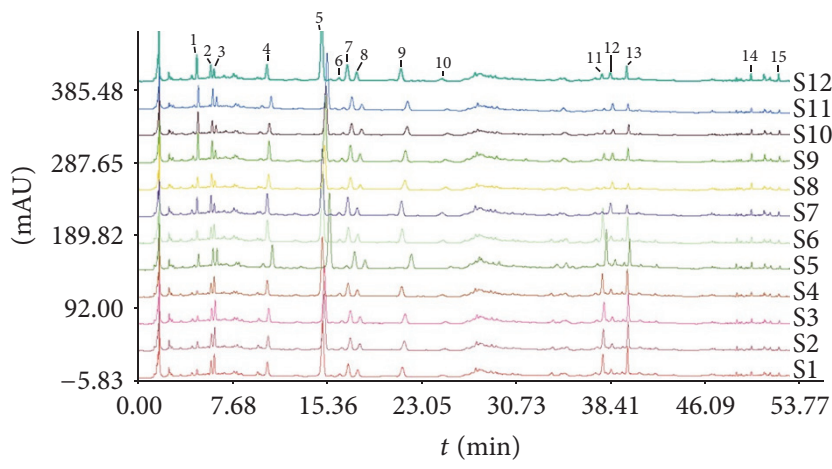

FIGURE 1: UPLC fingerprints of 15 batches of Danlou Tablets. 2: 5hydroxymethyl furfural; 3: danshensu; 5: puerarin; 9: daidzin; 11: Sal B; 13: Sal A; and 15: tanshinone II A.

$30 \%$ of patients undergoing PCI therapy developed PMI, which is significantly associated with bad long-term prognosis $[1,2]$.

Clinical trials have revealed the efficacy of high-dose statin treatment in significantly reducing the incidence of PMI among patients after coronary revascularization [35]. However, high-dose statin administration has been associated with severe side effects, including increased risk of new-onset diabetes, liver damage, rhabdomyolysis, and intracerebral haemorrhage [6-8]. Therefore, recent studies endeavoured to discover alternative natural agents that could reduce the incidence of myocardial necrosis after coronary intervention with low risk of side effects $[9,10]$.

Over the past several years, there has been a surge of interest in the use of Chinese medicine for alleviating cardiovascular diseases, including angina pectoris, myocardial infarction, and chronic heart failure [11-13]. According to the theory of Traditional Chinese Medicine (TCM), the primary cause of coronary heart disease is intermingled phlegm and blood stasis. The Danlou Tablet, a patented Chinese medicine, has been approved by China Food and Drug Administration for patients with coronary heart disease and angina pectoris in 2005. The Danlou Tablet has been demonstrated to significantly alleviate phlegm and stasis mutual obstruction, decrease the serum level of inflammation molecular, and improve the quality of life in patients with unstable angina pectoris [14]. Furthermore, basic research has shown that it could decrease the area of myocardial ischemia and increase ion transport channel-related enzyme activities for the arrhythmia model rats induced by transient myocardial ischemia/reperfusion [15]. Ultraperformance liquid chromatography-tandem mass spectrometer (UPLCMS/MS) was also used to analyse 15 quality-control markers of Danlou Tablet, and good consistency of the active markers was found among 12 different batches (Figure 1).

In the current study, we evaluated the hypothesis that Danlou Tablet treatment in patients with non-st-segment elevation acute coronary syndromes (NSTE-ACS) undergoing PCI would decrease the incidence of PMI and improve the clinical outcome by a multicentre, randomized, prospective, double-blind, placebo-controlled trial.

\section{Methods}

2.1. Study Design. The protocol of this clinical trial has been revealed in detail previously [16]. Briefly, the study followed the Declaration of Helsinki and was approved by Institutional Ethics Committee of Guangdong Provincial Hospital of Traditional Chinese Medicine and participating hospitals (Acknowledgments Section). Statin-naïve patients aged $\geq 18$ years with non-ST-segment elevation ACS including unstable angina or non-ST-segment elevation myocardial infarction (NSTEMI) with selective coronary angioplasty to be undertaken within $72 \mathrm{~h}$ of admission were screened. Patients with acute ST-segment elevation myocardial infarction (STEMI), cardiac shock, severe heart failure (left ventricular ejection fraction $<30 \%$ ), or hepatic dysfunction with elevated alanine aminotransferase and aspartate aminotransferase serum levels for any reason were excluded from this study. Written informed consent was obtained from each participating patient.

2.2. Interventions. Eligible patients were randomized to either the experimental group or the control group. Patients in the experimental group received the treatment of Danlou Tablet (4.5g per day for 48 hours before PCI and a further $4.5 \mathrm{~g}$ per day for 90 days after PCI). Patients in the control group received the administration of placebo. All enrolled patients received standard management care in accordance with the Guidelines for the Management of Patients with Unstable Angina/NSTEMI [17], including anti-platelet agents, anticoagulation agents, lipid-lowering agents, antiventricular remodelling, or antihypertensive therapy depending on the conditions of patients, irrespective of the randomization assignment. In particular, atorvastatin served as the unique lipid-lowering agent in the study, administrated with the moderate-intensity dose of $10 \mathrm{mg} /$ day before and after PCI without any loading doses for the purpose of minimising the effect of statin on the results.

Enrolled patients were assessed at $48 \mathrm{~h}$ before PCI, $8 \mathrm{~h}$ and $24 \mathrm{~h}$ after PCI, and 30 days and 90 days after PCI. Assessments included physical examination, vital signs, quality-oflife measurement, electrocardiogram (ECG), and echocardiography. Furthermore, blood samples were collected for laboratory tests, including cardiac biomarkers (troponin I and creatine kinase-myocardial band [CK-MB]), lipids, biochemistry, haematology, C-reactive protein (CRP), and urinalysis.

2.3. Randomization and Blinding. Participants were randomly assigned into two groups. Randomization was stratified by centre with permuted block size. The randomized sequence was generated by computer with SAS 9.2 software (SAS institute Inc., Cary, USA) and saved in China-Australia International Research Centre for Chinese Medicine (CAIRC-CM), Guangdong Academy of Chinese Medical Sciences. Allocation of treatments was distributed by sealed, opaque, and numbered envelopes. Practitioners and participants were unaware of their assignment. One statistician who generated the blinding code was aware of the drug allocation. Participants, physicians, outcome assessors, and 
other statisticians and practitioners, however, remained blind to treatment assignments before the results were revealed. The placebo was prepared and packed by a pharmaceutical company (Jilin Connell Pharmaceutical Co. Ltd., China). The placebo was designed similarly to Danlou Tablet in shape, size, and taste. Each participant was provided with a bottle of water labelled with their unique number according to randomization.

2.4. Outcomes. The primary endpoints of this trial were the incidence of death, nonfatal myocardial infarction, target vessel revascularization (bypass surgery or repeat PCI), and rehospitalization due to acute cardiovascular events (severe angina or heart failure) within 30 days of the procedure. Secondary endpoints included the incidence of major adverse cardiovascular events (MACEs) within 90 days of PCI, the proportion of patients with elevated biomarkers of myocardial injury (troponin I) at $8 \mathrm{~h}$ and $24 \mathrm{~h}$ after PCI, and the proportion of patients with elevated CRP level at these time points. Nonfatal myocardial infarctions included spontaneous myocardial infarction, PMI, and myocardial infarctions related to stent thrombosis. PMI was defined as a postprocedural increase of cardiac troponin I (cTn I) values more than $5 \times 99$ th percentile of the upper reference limit (URL) or a rise of cTn I values $>20 \%$ if baseline values were elevated. Spontaneous myocardial infarctions were considered to be related to ischemia due to plaque erosion and/or rupture, fissuring, or dissection [18].

Furthermore, the safety and tolerability of experimental drugs were evaluated by the incidence, severity, and relationship to treatment of adverse events (AEs), including clinically significant changes in vital signs, physical examination findings, and laboratory measurements.

2.5. Sample Size Estimation. The sample size was calculated based on the reduction of incidence of MACE by a twosided test with level size of $5 \%$ and a power of $80 \%$ chance of detecting a difference. A previous trial revealed the incidence of MACE as $17 \%$ in patients treated with low-dose statin therapy within 30 days after PCI [3]. Assuming a MACE incidence of $5 \%$ in the experimental group, it was calculated that a minimum of 99 patients would be required for each group. Additionally, we estimated that up to $10 \%$ of initial participants may withdraw from the trial (PASS 11.0 software, NCSS, Utah, USA). We thus determined that 232 eligible cases would be required.

2.6. Statistical Analysis. The trial database was blindly reviewed before the data were locked and unblended by the independent data collection centre, China-Australia International Research Centre for Chinese Medicine. Data from all participants who underwent randomization were analysed based on the intention-to-treat (ITT) principle. A per-protocol (PP) analysis was performed to test the robustness of the trial results. Baseline clinical and demographic characteristics were expressed as mean $\pm \mathrm{SD}$, median, or interquartile range (IQR). For comparisons, two samples were compared by $t$-test for normally distributed values; otherwise the Mann-Whitney $U$ test was used. Proportions were analysed using the Chi-square test or the exact test method. Kaplan-Meier plots of cumulative incidence freedom of overall MACEs were constructed from the 30-day and 90-day follow-up. For all analyses, a value of $p<0.05$ (twotailed) was considered statistically significant. All calculations were conducted using SPSS software version 18.0 (IBM Inc., New York, USA).

\section{Results}

3.1. Patient Population. Between November 25, 2012, and March 23, 2014, 340 patients were screened: among them, 60 were excluded because of previous or current treatment with statins; 35 were excluded because they required an emergency PCI approach; 21 were excluded because of severe heart failure with ejection fraction $<30 \%$; and 5 were excluded because of contraindications to statin treatment (e.g., hepatic dysfunction or serious adverse reaction) (Figure 2). Eligible patients $(n=219)$ received the study assignment drug (Danlou Tablet or placebo) before PCI. Procedural success was obtained in all patients; 4 patients ( 2 in each group) had no-reflow or slow-flow phenomenon, which was significantly alleviated following intracoronary administration of platelet glycoprotein IIb/IIIa inhibitors (tirofiban). The last subject completed follow-up in June, 2014. Data entry was completed by November, 2014.

Demographic and clinical features of patients in the Danlou Tablet group and placebo group are indicated in Table 1. Patient characteristics were not significantly different in age, gender, cardiovascular risk factors, concomitant diseases, clinical presentation, cardiac function, blood creatinine levels, and medical therapy at the time of intervention. In comparing the main procedural features treatments in both the Danlou Tablet and placebo groups, there were statistically significant differences in the stent length (indicated in Table 2), but there were no statistically significant differences in coronary anatomy, multivessel lesion type, procedural characteristics of intervention, number of stents per patient, or the diameter of implanted stents $(p>0.05)$.

3.2. Cardiovascular Events. The primary endpoint was assessed at 30 days after coronary revascularization (Table 3). There was no statistically significant difference in the incidence of MACE in the Danlou Tablet group (22.0\% of patients [24 of 109]) in the Danlou Tablet group compared to the placebo group (33.6\% of patients [37 of 110]) $(p=0.06)$. At 90 days after PCI, however, the incidence of MACE in the Danlou Tablet group was significantly lower than in the placebo group (23.9\% versus $37.3 \%, p=0.03$, Table 4$)$. Furthermore, Kaplan-Meier curves showed that MACE-free survival rates at the 90-day follow-up were significantly greater in Danlou Tablet group than in the placebo group $(p=0.04$, Figure 3$)$. The lower rate of MACE at 90 days in the Danlou Tablet group was mostly driven by a reduced incidence of nonfatal myocardial infarction (22\% versus $34.5 \%, p=0.04$ ). The vast majority of nonfatal myocardial infarction cases occurred within 24 hours after PCI and were defined as PMIs (Figure 4). One patient (0.9\%) in the placebo group died due to probable stent thrombosis complicated 


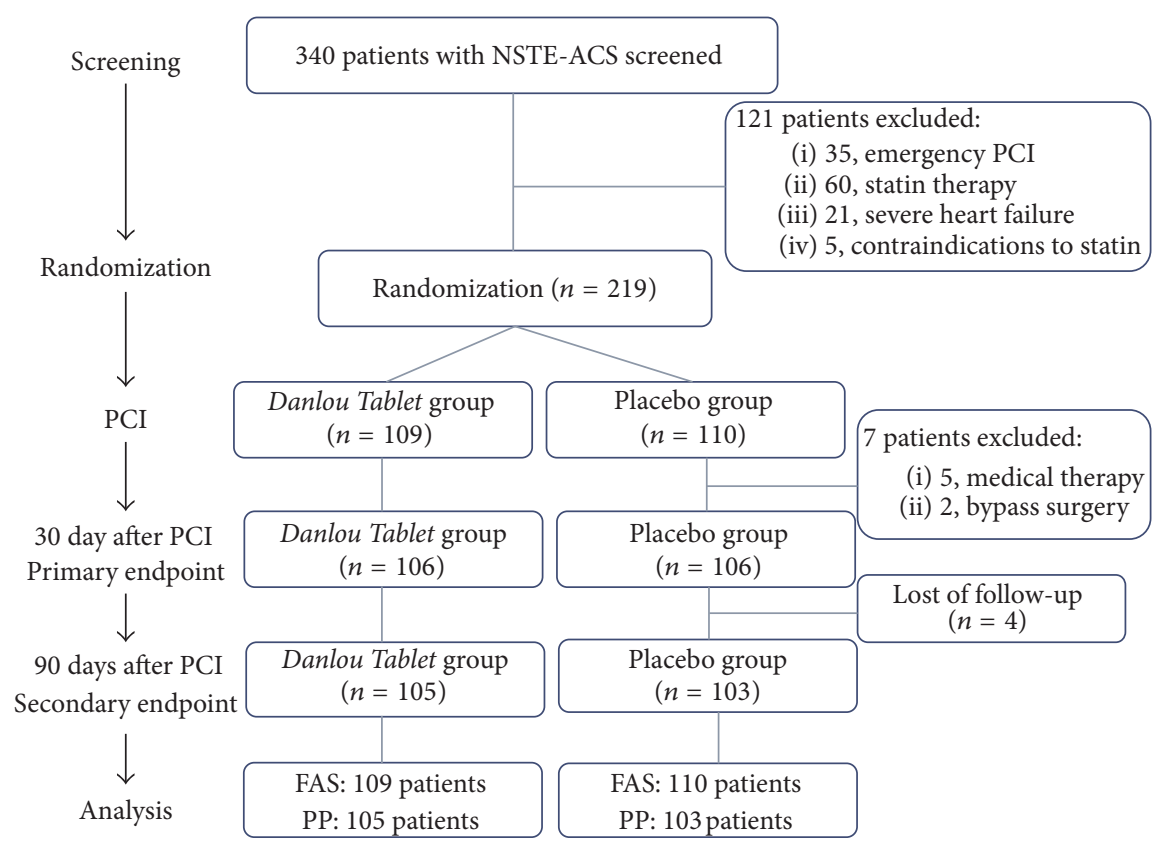

FIGURE 2: Flow diagram of Danlou Tablet for patients with ACS undergoing PCI. FAS, full-analysis set; NSTE-ACS, non-ST-segment elevation acute coronary syndrome; PCI, percutaneous coronary intervention; and PP, per protocol.

TABLE 1: Main demographic and clinical features of the two groups.

\begin{tabular}{|c|c|c|c|c|}
\hline Variable & Danlou Tablet group $(n=109)$ & Placebo group $(n=110)$ & $\chi^{2}(Z)$ & $p$ value \\
\hline Age, yr & $62.89 \pm 9.23$ & $63.89 \pm 10.03$ & 0.04 & 0.44 \\
\hline Men, number (\%) & $72(66.1)$ & $74(67.3)$ & 0.04 & 0.85 \\
\hline Body weight, kg & $66.91 \pm 10.14$ & $66.65 \pm 9.54$ & -0.6 & 0.55 \\
\hline Height, cm & $166.41 \pm 6.85$ & $166.00 \pm 8.17$ & -0.23 & 0.82 \\
\hline Family history of coronary disease, number (\%) & $18(16.5)$ & $10(9.1)$ & 2.71 & 0.10 \\
\hline \multicolumn{5}{|l|}{ Concomitant diseases, number (\%) } \\
\hline Previous coronary heart disease & $41(37.6)$ & $47(42.7)$ & 0.60 & 0.44 \\
\hline Previous coronary intervention & $9(8.3)$ & $17(15.5)$ & 2.71 & 0.10 \\
\hline Systemic hypertension & $51(46.8)$ & $63(57.3)$ & 2.41 & 0.12 \\
\hline Diabetes mellitus & $21(19.3)$ & $19(17.3)$ & 0.15 & 0.70 \\
\hline Dyslipidemia & $38(34.9)$ & $38(34.5)$ & 0.002 & 0.96 \\
\hline Heart failure & $1(0.9)$ & $4(3.6)$ & & $0.37^{*}$ \\
\hline Arrhythmia & $4(3.7)$ & $10(9.1)$ & 2.69 & 0.10 \\
\hline Stroke & $7(6.4)$ & $8(7.3)$ & 0.06 & 0.80 \\
\hline Current smoker, number (\%) & $47(43.1)$ & $44(40.0)$ & 0.22 & 0.64 \\
\hline Cardiac Marker Elevation, number (\%) & $30(27.5 \%)$ & $33(30.0 \%)$ & 0.16 & 0.69 \\
\hline \multicolumn{5}{|l|}{ Clinical pattern, number (\%) } \\
\hline Unstable angina & $88(80.7)$ & $87(79.1)$ & \multirow{2}{*}{0.09} & \multirow{2}{*}{0.76} \\
\hline NSTEMI & $21(19.3)$ & $23(20.9)$ & & \\
\hline \multicolumn{5}{|l|}{ Cardiac function, number (\%) } \\
\hline Level I & $41(37.6)$ & $41(37.3)$ & \multirow{3}{*}{0.03} & \multirow{3}{*}{0.99} \\
\hline Level II & $53(48.6)$ & $53(48.2)$ & & \\
\hline Level III & $15(13.8)$ & $16(14.5)$ & & \\
\hline
\end{tabular}

Values are given as number of patients (\%) or mean \pm SD. NSTEMI, non-ST-segment elevation myocardial infarction; PCI, percutaneous coronary intervention; $\mathrm{ACE}$, angiotensin-converting enzyme; and ARB, angiotensin II receptor blocker. ${ }^{*} p$ value is from Fisher's exact test. 
TABLE 2: Angiographic and procedural features of the two groups.

\begin{tabular}{|c|c|c|c|c|}
\hline Variable & Danlou Tablet group $(n=109)$ & Placebo group $(n=110)$ & $\chi^{2}(Z)$ & $p$ value \\
\hline Multivessel coronary artery disease, number (\%) & $62(56.9)$ & $63(57.3)$ & 0.00 & 0.95 \\
\hline \multicolumn{5}{|l|}{ Vessel treated, number (\%) } \\
\hline Left main & $2(1.8)$ & $2(1.8)$ & & $1.00^{*}$ \\
\hline Left anterior descending & $73(67.0)$ & $65(59.1)$ & 1.46 & 0.23 \\
\hline Left circumflex & $26(23.9)$ & $34(30.9)$ & 1.37 & 0.24 \\
\hline Right coronary artery & $35(32.1)$ & $30(27.3)$ & 0.61 & 0.43 \\
\hline Restenotic lesions, number (\%) & $1(0.9)$ & $1(0.9)$ & & 1.00 \\
\hline Multivessel intervention, number (\%) & $24(22.0)$ & $18(16.4)$ & 1.13 & 0.29 \\
\hline \multicolumn{5}{|l|}{ Type of intervention, number (\%) } \\
\hline Balloon only & $1(0.9)$ & $1(0.9)$ & & $1.00^{*}$ \\
\hline Stent & $108(99.1)$ & $109(99.1)$ & & $1.00^{*}$ \\
\hline Number of stents per patient & $1.49 \pm 0.70$ & $1.41 \pm 0.67$ & -0.89 & 0.37 \\
\hline Stent diameter, $\mathrm{mm}$ & $2.94 \pm 0.43$ & $2.92 \pm 0.43$ & -0.87 & 0.39 \\
\hline Total stent length, $\mathrm{mm}$ & $20.52 \pm 7.01$ & $22.37 \pm 7.46$ & -2.34 & 0.02 \\
\hline
\end{tabular}

Values are given as number of patients (\%) or mean \pm SD. ${ }^{*} p$ value is from Fisher's exact test.

TABle 3: MACE at 30 days after PCI in the Danlou Tablet and placebo groups.

\begin{tabular}{|c|c|c|c|c|}
\hline & \multicolumn{2}{|c|}{ Incidence } & \multicolumn{2}{|l|}{ Treatment difference (\%) } \\
\hline & Danlou Tablet group & Placebo group & Difference $^{\mathrm{a}}$ of incidence $(95 \% \mathrm{CI})$ & $p$ value \\
\hline Full-analysis set & $N=109$ & $N=110$ & & \\
\hline Total MACE, $n(\%)$ & $24(22.0)$ & $37(33.6)$ & $1.8(1.0,3.3)$ & $0.06^{\mathrm{c}}$ \\
\hline Cardiac death, $n(\%)$ & 0 & 0 & & \\
\hline Nonfatal MI, $n(\%)$ & $24(22.0)$ & $37(33.6)$ & $1.8(1.0,3.3)$ & $0.06^{\mathrm{c}}$ \\
\hline Target vessel revascularization, $n(\%)$ & 0 & $1(0.9)$ & & $1.0^{\mathrm{d}}$ \\
\hline Rehospitalization due to $\mathrm{CVE}^{\mathrm{b}}, n(\%)$ & 0 & 0 & & \\
\hline Per-protocol analysis set & $N=107$ & $N=109$ & & \\
\hline Total MACE, $n(\%)$ & $24(22.4)$ & $36(33.0)$ & $1.7(0.9,3.1)$ & $0.08^{\mathrm{c}}$ \\
\hline Cardiac death, $n(\%)$ & $0(0)$ & $0(0)$ & & \\
\hline Nonfatal myocardial infarction, $n(\%)$ & $24(22.4)$ & $36(33.0)$ & $1.7(0.9,3.1)$ & $0.08^{c}$ \\
\hline Target vessel revascularization, $n(\%)$ & 0 & $1(0.9)$ & & $1.0^{\mathrm{d}}$ \\
\hline Rehospitalization due to $\mathrm{CVE}^{\mathrm{b}}, n(\%)$ & 0 & 0 & & \\
\hline
\end{tabular}

CI, confidence interval; CVE, cardiovascular events; MACE, major adverse cardiac event; and MI, myocardial infarction.

${ }^{\mathrm{a}}$ Difference of incidence $=$ Danlou Tablet - placebo.

${ }^{\mathrm{b}}$ Cardiovascular events included severe angina or heart failure (NYHF $\left.\geqslant I V\right)$.

${ }^{c} p$ value is from the continuity-adjusted Chi-square test.

${ }^{\mathrm{d}} p$ value is from Fisher's exact test.

with cardiac shock. In two patients $(1.8 \%)$ in the Danlou Tablet group and five patients (4.5\%) in the placebo group, instent restenosis occurred, and coronary revascularization had to be repeated. There was no statistically significant difference in the incidences of target vessel revascularization between the treated and placebo groups $(p=0.45)$.

3.3. Biomarker Results. Before the procedure, there were no significant differences in the percentage of patients with abnormal cTn I elevation between two group (27.5\% versus $30.0 \%, p=0.69$, Table 1). After the procedure, 8 and $24 \mathrm{hr}$ post-PCI cTn I $>3 \times 99$ th percentile of URL occurred more frequently in the placebo arm than in the Danlou Tablet group (25.7\% versus $40.9 \%, p=0.02$, and $30.3 \%$ versus $41.8 \%$, $p=0.08$ ) (Figure 5(a)). Moreover, the proportion of patients with elevated levels of cTn I $>5 \times 99$ th percentile of URL was significantly lower in the Danlou Tablet group at $8 \mathrm{~h}(22.0 \%$ versus $35.4 \%, p=0.04)$ and $24 \mathrm{~h}$ after PCI (23.9\% versus $38.2 \%, p=0.02$ ) (Figure 5(b)). Analyses on the population with elevated CRP levels showed no statistically significant differences between the Danlou Tablet group and the placebo group at $8 \mathrm{~h}(37.1 \%$ versus $32.0 \%, p=0.44)$ and $24 \mathrm{~h}(47.6 \%$ versus $42.7 \%, p=0.48$ ) after PCI.

3.4. Safety Analysis. Only two patients in Danlou Tablet group had minor gastrointestinal adverse events (nausea and diarrhea), which were relieved after two days of suspension in the management of the experimental drug. Meanwhile, there were no significant ECG or physical examination findings or changes in laboratory parameters potentially associated with experimental drugs. 
TABLE 4: MACE at 90 days after PCI in the Danlou Tablet and placebo groups.

\begin{tabular}{|c|c|c|c|c|}
\hline & \multicolumn{2}{|c|}{ Incidence } & \multicolumn{2}{|l|}{ Treatment difference (\%) } \\
\hline & Danlou Tablet group & Placebo group & Difference of incidence (95\% CI) & $p$ value \\
\hline Full-analysis set & $N=109$ & $N=110$ & & \\
\hline Total MACE, $n(\%)$ & $26(23.9)$ & $41(37.3)$ & $1.9(1.1,3.4)$ & $0.03^{\mathrm{c}}$ \\
\hline Cardiac death, $n(\%)$ & 0 & $1(0.9)$ & & $1.00^{\mathrm{d}}$ \\
\hline Nonfatal MI, $n(\%)$ & $24(22.0)$ & $38(34.5)$ & $1.9(1.0,3.4)$ & $0.04^{\mathrm{c}}$ \\
\hline Target vessel revascularization, $n(\%)$ & $2(1.8)$ & $5(4.5)$ & $2.5(0.5,13.4)$ & $0.45^{\mathrm{d}}$ \\
\hline Rehospitalization due to $\mathrm{CVE}^{\mathrm{b}}, n(\%)$ & 0 & 0 & & \\
\hline Per-protocol analysis set & $N=105$ & $N=103$ & & \\
\hline Total MACE, $n(\%)$ & $25(23.8)$ & $35(34.0)$ & $1.6(0.9,3.0)$ & $0.11^{\mathrm{c}}$ \\
\hline Cardiac death, $n(\%)$ & 0 & $1(1.0)$ & & $0.50^{\mathrm{d}}$ \\
\hline Nonfatal MI, $n(\%)$ & $23(21.9)$ & $32(31.1)$ & $1.6(0.9,3.0)$ & $0.13^{\mathrm{c}}$ \\
\hline Target vessel revascularization, $n(\%)$ & $2(1.9)$ & $5(4.9)$ & $2.6(0.5,13.9)$ & $0.28^{\mathrm{d}}$ \\
\hline Rehospitalization due to $\mathrm{CVE}^{\mathrm{b}}, n(\%)$ & 0 & 0 & & \\
\hline
\end{tabular}

$\mathrm{CI}$, confidence interval; CVE, cardiovascular events; MACE, major adverse cardiac event; and MI, myocardial infarction.

${ }^{a}$ Difference of incidence $=$ Danlou Tablet - placebo.

${ }^{\mathrm{b}}$ Cardiovascular events included severe angina or heart failure (NYHF $\geqslant$ IV).

${ }^{c} p$ value is from the continuity-adjusted Chi-square test.

${ }^{\mathrm{d}} p$ value is from Fisher's exact test.

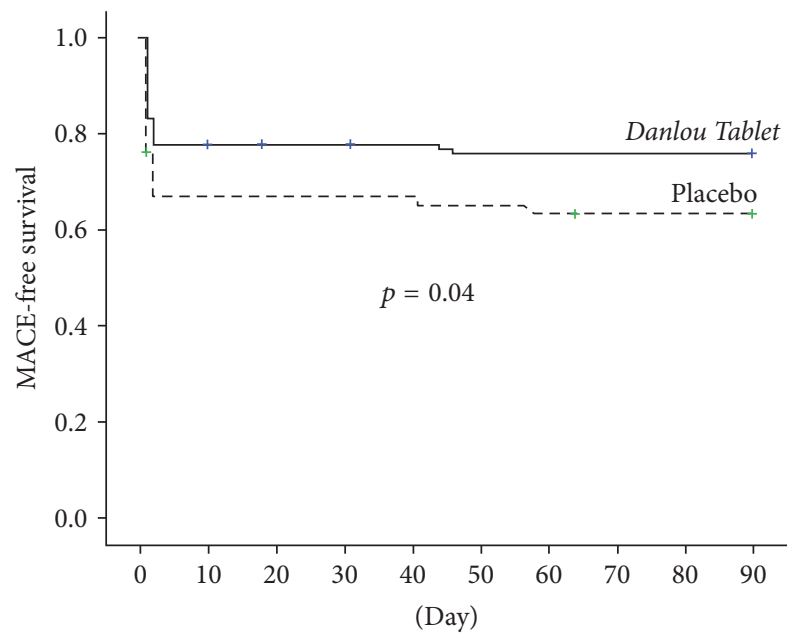

FIgURE 3: Survival curves. Kaplan-Meier curves of 90-day major adverse cardiac event- (MACE-) free survival in the 2 arms. MACE, major adverse cardiac event (death, nonfatal myocardial infarction, target vessel revascularisation, and rehospitalization due to acute cardiovascular events).

\section{Discussion}

It has been reported that a large number of patients undergoing PCI might suffer from myocardial injuries arising from the procedure itself, which is significant enough to serve as a negative prognostic factor [19]. Although previous studies have shown that pretreatment with high-dose statin is associated with reduced incidence of PMI in patients with acute coronary events $[3,4]$, still more studies did not demonstrate similar results $[20,21]$. Particularly, a trial in which statinnaïve Korean and Chinese patients with NSTE-ACS received additional loading high-dose atorvastatin before PCI showed

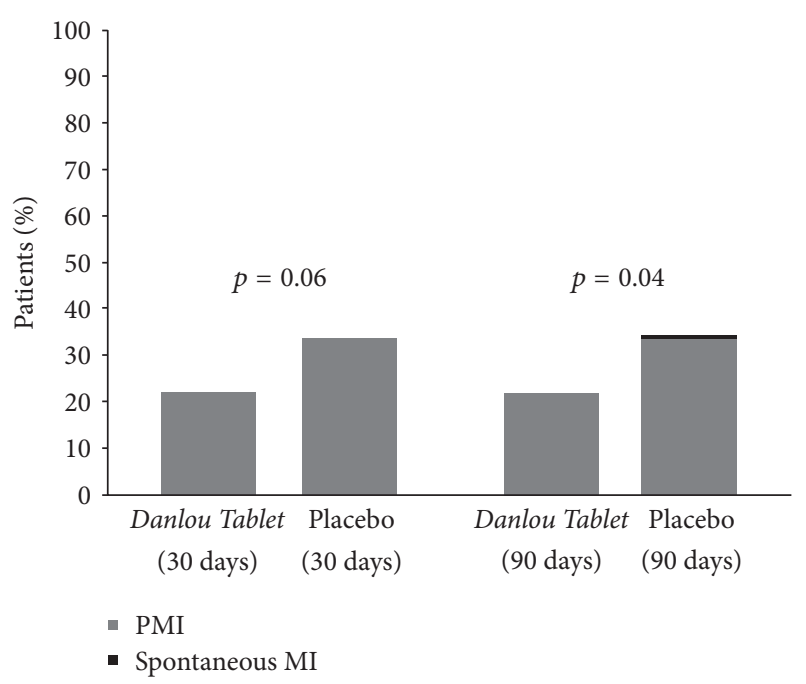

FIGURE 4: Non-fatal MI. * PMI: periprocedure myocardial infarction.

that there were no benefits compared with usual post-PCI atorvastatin treatment [21]. Moreover, recent trials revealed that there were no expected benefits of high-intensity statin treatments in East Asian patients compared with moderateor low-intensity statin treatments $[22,23]$. The reason why there were the different benefits of high-dose statin between Asian and Caucasians population could be attributed to the race difference of statin pharmacokinetics. Therefore, strong evidence-based natural agents to improve East Asian patient management and reduce the incidence of PMI are critical to address this challenge.

The present study was a multicentre, randomized controlled trial conducted in a statin-naïve Chinese ACS population undergoing selective PCI. Herein, we compare 


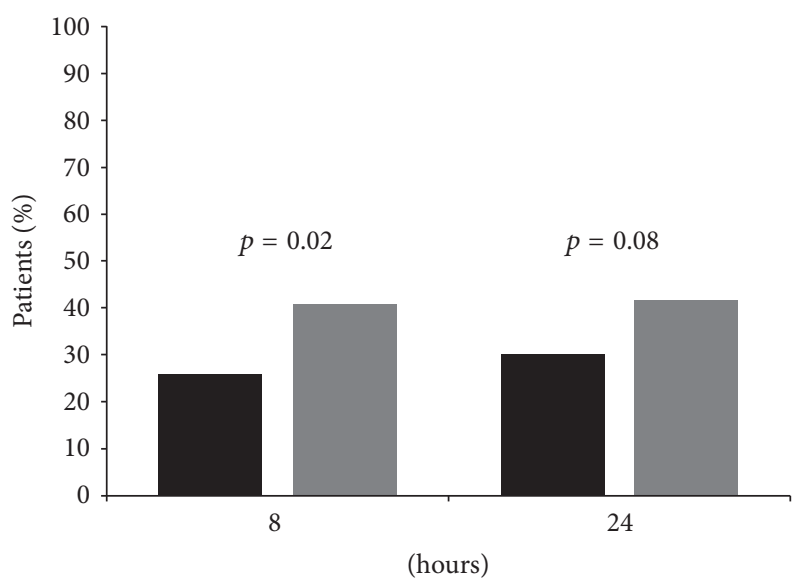

- Danlou Tablet

- Placebo

(a)

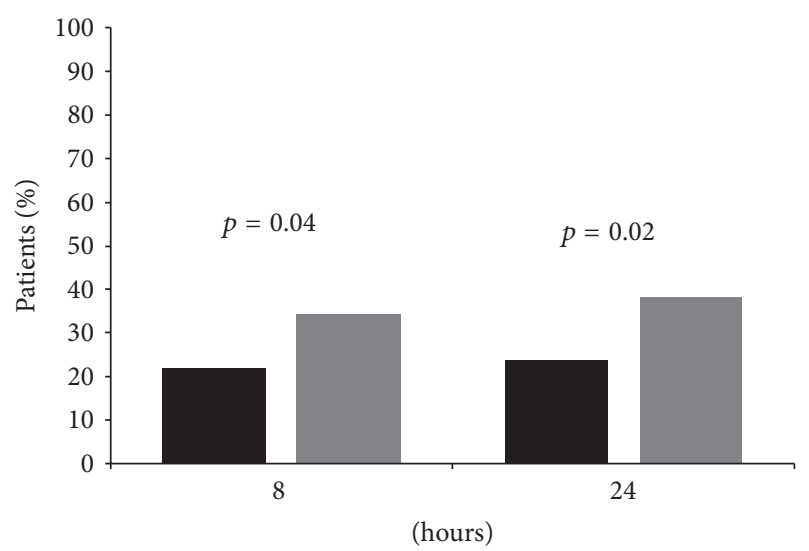

- Danlou Tablet

- Placebo

(b)

FIgURE 5: Cardiac Marker Elevations. (a) TnI $>3 \times 99$ th percentile of the upper reference limit (URL) at 8 and 24 hours after PCI in the Danlou Tablet group and in the placebo group. (b) TnI $>5 \times 99$ th percentile of the URL at 8 and 24 hours after PCI in the Danlou Tablet group and in the placebo group.

a group in which Danlou Tablet was administered for 2 days before elective PCI following a further treatment with the control group who received placebo based on usual care including moderate-intensity statin treatment (atorvastatin, $10 \mathrm{mg} /$ day). Our study demonstrated that the proportion of patients with elevated levels of troponin I was significantly lower in the Danlou Tablet group at $8 \mathrm{~h}$ and $24 \mathrm{~h}$, suggesting that Danlou Tablet pretreatment was associated with a significantly lower occurrence of PMI (Figure 5(b)). There was a tendency towards a lower occurrence of major cardiac events 30 days after PCI in patients with ACS undergoing selective interventional therapy $(22.0 \%$ versus $33.6 \%, p=0.06)$. The MACE-free Kaplan-Meier curves showed a significantly better event-free survival at 90 days in the Danlou Tablet arm. This change may be attributed to a significant reduction of nonfatal myocardial infarctions. The incidence of PMI in our trial was higher than that in the ARMYDAR-ACS [4] or ROMA trials [5]. The different diagnostic criteria of PMI may have led to this result; in the latter trials, PMI was diagnosed by the increase of creatine kinase-MB instead of cTn I.

The predominant mechanisms of PMI are complicated, including vulnerable plaque disruption [24], ischemia/reperfusion injury [25], oxidative stress, platelet activation [26], and inflammatory cytokines activation [27] induced by balloon pressure inflation or stent implantation during the procedure. The Danlou Tablet is a potent herbal compound mainly consisting of Salvia, Ligusticum chuanxiong Hort, Trichosanthes kirilowii, and Allium macrostemon. Chen et al. [28] report that Danlou Tablet may accelerate blood circulation and eliminate intravascular phlegm, which have critical roles in managing the ischemic disease based on the Traditional Chinese Medicine (TCM) theory. An increasing number of studies have been conducted to elucidate the cardioprotective effects of Danlou Tablet. Liu et al. investigated the efficacy of Danlou Tablet in improving cardiac function in miniature swine with experimental coronary disease and found that the Danlou Tablet treatment significantly improved cardiac function and histopathology and downregulated malondialdehyde (MDA) and superoxide dismutase (SOD) serum levels [29]. Guo et al. examined the effects of Danlou Tablet on arrhythmia model rats induced by transient myocardial ischemia/reperfusion (I/R) and revealed that Danlou Tablet inhibited the incidence of fatal and nonfatal ventricular fibrillation. The reduced frequency and duration of ventricular tachycardia might be related to lowering the degree of myocardial ischemia and increasing ion transport channelrelated enzyme activities $\left(\mathrm{Na}^{+}-\mathrm{K}^{+}\right.$ATPase and CaATPase) [15]. Similarly, our recent studies also suggested that administration of Danlou Tablet could alleviate the severity of riskregion myocardial ischemia and reperfusion arrhythmia in vivo [30]. Moreover, recent studies revealed that puerarin, the main component of Danlou Tablet by UPLC-MS/MS analysis (Figure 1), could significantly protect cardiomyocytes from anoxia/reoxygenation injury in vitro $[31,32]$.

In addition, endothelial injury induced by balloon or stent inflating is another key contributor for PMI [33]. Yang et al. found that the original superfine Danlou Tablet improved endothelial function in rats with arterial endothelial injury by downregulating the cardiac chymase signal pathway and chymase-mediated angiotensin II production [34]. Hong's study suggested that Danlou Tablet may protect vascular endothelium and improve formation of new capillaries on the edges of the ischemic myocardial zones, thereby contributing to the ultimate reduction of the infarct size and subsequent alleviation of the progressive heart failure [35]. Furthermore, another possible mechanism underlying the beneficial effects of Danlou Tablet may involve the alleviation of inflammatory reactions at the atherosclerotic plaque [28]. A clinical trial by Wang et al. showed the treatment of Danlou Tablet could alleviate inflammatory activation by reducing the serum level of hs-CRP, soluble CD40, and interleukin-6 [14]. However, our study demonstrated that there was no significant difference in the serum level of hs-CRP between two arms. Consequently, the anti-inflammation effects of this patented Chinese herb 
need to be further explored by more studies in vitro and in vivo.

Therefore, the benefits of Danlou Tablet in reducing PMI could be explained by the reported multiple mechanisms by which the treatment or its components inhibit ischemia reperfusion injury, decrease oxidative stress responses, and improve endothelial dysfunction and microvessel coronary circulation. Pre-PCI treatment of Danlou Tablet in NSTEACS patients may consequently translate into clinical positive outcomes.

\section{Limitations}

This study has some limitations. Firstly, the figures we used to estimate the sample size were not consistent with the actual results, which led to relatively lower power than expected. Secondly, biochemical measurements (e.g., CRP and cTn I) were not performed in the laboratory, which may have decreased the reliability of results. Furthermore, this trial was undertaken in China; whether the effects of the trial drugs would be similar in other ethnic groups is unknown.

\section{Conclusion}

In conclusion, the present trial implied that treatment with Danlou Tablet based on the low-dose of statin could reduce the incidence and magnitude of PMI and subsequently improve clinical outcomes in patients receiving elective revascularization with unstable angina and non-ST-segment elevation acute coronary syndromes. If confirmed by larger additional randomized studies, these findings may support the indication of "upstream" administration of this alternative natural agent in patients with acute coronary syndromes treated with an early invasive strategy.

$\begin{array}{ll}\text { Abbreviations } \\ \text { ACS: } & \text { Acute coronary syndrome } \\ \text { CHD: } & \text { Coronary heart disease } \\ \text { CK-MB: } & \text { Creatine kinase isoenzyme MB } \\ \text { CMR: } & \text { Cardiac magnetic resonance } \\ \text { CRP: } & \text { C-reactive protein } \\ \text { cTn I: } & \text { Cardiac troponin I } \\ \text { DSMB: } & \text { Data and Safety Monitoring Board } \\ \text { MACE: } & \text { Major adverse cardiac event } \\ \text { NYHA: } & \text { New York Heart Association } \\ \text { NSTE-ACS: } & \text { Non-ST elevation acute coronary } \\ & \text { syndrome } \\ \text { PMI: } & \text { Periprocedural myocardial injury } \\ \text { PCI: } & \text { Percutaneous coronary intervention. }\end{array}$

\section{Competing Interests}

The authors declare that they have no conflict of interests.

\section{Authors' Contributions}

Lei Wang, Xujie Zhao, and Shuai Mao contributed equally to this article. Lei Wang and Shuai Mao drafted this manuscript;
Lei Wang and Minzhou Zhang designed the described study; Xujie Zhao and Shaonan Liu completed the statistical analysis; Xinfeng Guo designed the randomization and concealment for this study; Lei Wang, Liheng Guo, Tinghai Du, Fuhai Zhao, Keng Wu, Hongliang Cong, and Yang Wu performed this study; Keji Chen and Phillip C. Yang made critical revision of the manuscript and contributed to the rationalization of the study. All authors read and approved the final manuscript.

\section{Acknowledgments}

This work was financially supported jointly by grants (no. 2012A032500013 and no. 2014A020221037, to Lei Wang) from the Department of Science and Technology and the Academy of Traditional Chinese Medicine of Guangdong Province, grant (2015A030306049, to Lei Wang) from Guangdong Natural Science Funds for Distinguished Young Scholar, and grants (no. 81573708 and no. 81202782, to Lei Wang) from National Natural Science Foundation of China. The authors gratefully acknowledge the contributions of the following sites (and staff) who participated in this study: 2nd affiliated Hospital of Guangzhou University of Chinese Medicine (Liheng Guo, Lei Wang, Yuanshen Zhou, Yi Ren, Banghan Ding, Ningzhi Zhou, and Yi Su); Xiyuan Hospital of Chinese Academy of Traditional Chinese Medicine (Fuhai Zhao, Xin Wang); Yueyang Hospital of Integrated Medicine of Shanghai University of Traditional Chinese Medicine (Haiming Luo, Huanliang Jin); Oriental Hospital of Beijing University of Traditional Chinese Medicine (Yang Wu, Jie Cui); Chest Hospital of Tianjin (Hongliang Cong, Yuecheng $\mathrm{Hu}$, and Chunjie Li); 1st Affiliated Hospital of Henan College of Traditional Chinese Medicine (Tinghai Du, Peng Chen); the Affiliated Hospital of Guangdong Medical College (Keng Wu, Cheng Yang); Wuyi Hospital of Traditional Chinese Medicine of Jiangmen City (Haiyu Yang, Tao Yu, and Xinjun Zhao).

\section{References}

[1] G. G. Babu, J. M. Walker, D. M. Yellon, and D. J. Hausenloy, "Peri-procedural myocardial injury during percutaneous coronary intervention: an important target for cardioprotection," European Heart Journal, vol. 32, no. 1, pp. 23-31, 2011.

[2] M. D. Nettleman, L. Banitt, W. Barry, I. Awan, and E. E. I. Gordon, "Predictors of survival and the role of gender in postoperative myocardial infarction," American Journal of Medicine, vol. 103, no. 5, pp. 357-362, 1997.

[3] V. Pasceri, G. Patti, A. Nusca, C. Pristipino, G. Richichi, and G. Di Sciascio, "Randomized trial of atorvastatin for reduction of myocardial damage during coronary intervention: results from the ARMYDA (Atorvastatin for Reduction of MYocardial Damage during Angioplasty) study," Circulation, vol. 110, no. 6, pp. 674-678, 2004.

[4] G. Patti, V. Pasceri, G. Colonna et al., "Atorvastatin pretreatment improves outcomes in patients with acute coronary syndromes undergoing early percutaneous coronary intervention. Results of the ARMYDA-ACS randomized trial," Journal of the American College of Cardiology, vol. 49, no. 12, pp. 1272-1278, 2007.

[5] G. Sardella, G. Conti, M. Donahue et al., "Rosuvastatin pretreatment in patients undergoing elective PCI to reduce the 
incidence of myocardial periprocedural necrosis: the ROMA trial," Catheterization and Cardiovascular Interventions, vol. 81, no. 1, pp. E36-E43, 2013.

[6] D. Preiss, S. R. K. Seshasai, P. Welsh et al., "Risk of incident diabetes with intensive-dose compared with moderate-dose statin therapy: a meta-analysis," Journal of the American Medical Association, vol. 305, no. 24, pp. 2556-2564, 2011.

[7] J. Armitage, "The safety of statins in clinical practice," The Lancet, vol. 370, no. 9601, pp. 1781-1790, 2007.

[8] A. K. Pandit, P. Kumar, A. Kumar et al., "High-dose statin therapy and risk of intracerebral hemorrhage: a meta-analysis," Acta Neurologica Scandinavica, vol. 134, no. 1, pp. 22-28, 2016.

[9] G. Li, X. Zheng, H. Wang et al., "Multicenter investigation of compound danshen dripping pills on short-term clinical events in patient with ST elevation on myocardial infarction undergoing primary PCI (MICD-STEMIPCI)," Chinese Journal of Interventional Cardiology, vol. 19, no. 1, pp. 24-28, 2011.

[10] S. Mao, L. Wang, X. Zhao et al., "Sodium tanshinone IIA sulfonate for reduction of periprocedural myocardial injury during percutaneous coronary intervention (STAMP trial): rationale and design," International Journal of Cardiology, vol. 1, no. 182, pp. 329-333, 2015.

[11] H. Shang, J. Zhang, C. Yao et al., "Qi-shen-yi-qi dripping pills for the secondary prevention of myocardial infarction: a randomised clinical trial," Evidence-Based Complementary and Alternative Medicine, vol. 2013, Article ID 738391, 9 pages, 2013.

[12] L. Wang, M. Zhang, L. Guo et al., "Clinical pathways based on integrative medicine in chinese hospitals improve treatment outcomes for patients with acute myocardial infarction: a multicentre, nonrandomized historically controlled trial," Evidence-Based Complementary and Alternative Medicine, vol. 2012, Article ID 821641, 8 pages, 2012.

[13] X. Li, J. Zhang, J. Huang et al., "A multicenter, randomized, double-blind, parallel-group, placebo-controlled study of the effects of qili qiangxin capsules in patients with chronic heart failure," Journal of the American College of Cardiology, vol. 62, no. 12, pp. 1065-1072, 2013.

[14] S. H. Wang, J. Wang, and J. Li, "Efficacy assessment of treating patients with coronary heart disease angina of phlegm and stasis mutual obstruction syndrome by Danlou tablet," Zhongguo Zhong Xi Yi Jie He Za Zhi, vol. 32, no. 8, pp. 1051-1055, 2012.

[15] L.-L. Guo, J. Wang, F. Lin, and Y.-X. He, "Effect of danlou tablet on arrhythmia model rats induced by transient myocardial ischemia/ reperfusion," Zhongguo Zhong Xi Yi Jie He Za Zhi, vol. 34, no. 9, pp. 1125-1129, 2014.

[16] L. Wang, S. Mao, J.-Y. Qi et al., "Effect of Danlou Tablet (fftff) on peri-procedural myocardial injury among patients undergoing percutaneous coronary intervention for non-ST elevation acute coronary syndrome: a study protocol of a multicenter, randomized, controlled trial," Chinese Journal of Integrative Medicine, vol. 21, no. 9, pp. 662-666, 2015.

[17] J. L. Anderson, C. D. Adams, E. M. Antman et al., "ACCF/AHA focused update incorporated into the ACCF/AHA 2007 guidelines for the management of patients with unstable angina/nonST-elevation myocardial infarction: a report of the American College of Cardiology Foundation/American Heart Association Task Force on Practice Guidelines," Journal of the American College of Cardiology, vol. 61, no. 23, pp. 179-347, 2012.

[18] K. Thygesen, J. S. Alpert, A. S. Jaffe, M. L. Simoons, B. R. Chaitman, and H. D. White, "Third universal definition of myocardial infarction," Journal of the American College of Cardiology, vol. 60, no. 16, pp. 1581-1598, 2012.
[19] M. B. Nienhuis, J. P. Ottervanger, H. J. G. Bilo, B. D. Dikkeschei, and F. Zijlstra, "Prognostic value of troponin after elective percutaneous coronary intervention: a meta-analysis," Catheterization and Cardiovascular Interventions, vol. 71, no. 3, pp. 318324, 2008.

[20] J. Veselka, D. Zemánek, P. Hájek et al., "Effect of two-day atorvastatin pretreatment on the incidence of periprocedural myocardial infarction following elective percutaneous coronary intervention: a single-center, prospective, and randomized study," American Journal of Cardiology, vol. 104, no. 5, pp. 630633, 2009.

[21] Y. Jang, J. Zhu, J. Ge, Y.-J. Kim, C. Ji, and W. Lam, "Preloading with atorvastatin before percutaneous coronary intervention in statin-naïve Asian patients with non-ST elevation acute coronary syndromes: a randomized study," Journal of Cardiology, vol. 63, no. 5, pp. 335-343, 2014.

[22] Y.-F. Li, Q.-Z. Feng, W.-Q. Gao, X.-J. Zhang, Y. Huang, and Y.-D. Chen, "The difference between Asian and Western in the effect of LDL-C lowering therapy on coronary atherosclerotic plaque: a meta-analysis report," BMC Cardiovascular Disorders, vol. 15, no. 1, article 6, 2015.

[23] S.-P. Zhao, B.-L. Yu, D.-Q. Peng, and Y. Huo, "The effect of moderate-dose versus double-dose statins on patients with acute coronary syndrome in China: results of the CHILLAS trial," Atherosclerosis, vol. 233, no. 2, pp. 707-712, 2014.

[24] T. Kawamoto, H. Okura, Y. Koyama et al., "The relationship between coronary plaque characteristics and small embolic particles during coronary stent implantation," Journal of the American College of Cardiology, vol. 50, no. 17, pp. 1635-1640, 2007.

[25] M. K. Sinha, D. C. Gaze, J. R. Tippins, P. O. Collinson, and J. C. Kaski, "Ischemia modified albumin is a sensitive marker of myocardial ischemia after percutaneous coronary intervention," Circulation, vol. 107, no. 19, pp. 2403-2405, 2003.

[26] T. Cuisset, C. Frere, J. Quilici et al., "High post-treatment platelet reactivity is associated with a high incidence of myonecrosis after stenting for non-ST elevation acute coronary syndromes," Thrombosis and Haemostasis, vol. 97, no. 2, pp. 282287,2007

[27] N. Saleh, B. Svane, J. Jensen, L.-O. Hansson, M. Nordin, and P. Tornvall, "Stent implantation, but not pathogen burden, is associated with plasma C-reactive protein and interleukin-6 levels after percutaneous coronary intervention in patients with stable angina pectoris," American Heart Journal, vol. 149, no. 5, pp. 876-882, 2005.

[28] J. Chen, H. W. Cai, J. Miao, X. M. Xu, and W. Mao, "Danlou tablet fought against inflammatory reaction in atherosclerosis rats with intermingled phlegm and blood stasis syndrome and its mechanism study," Zhongguo Zhong Xi Yi Jie He Za Zhi, vol. 36, no. 6, pp. 703-708, 2016.

[29] J.-X. Liu, C.-R. Lin, J.-X. Ren et al., "Protective effect of formula of removing both phlegm and blood stasis on myocardial tissues of Chinese mini-swine with coronary heart disease of phlegmstasis cementation syndrome," Zhongguo Zhong Yao Za Zhi, vol. 39, no. 4, pp. 726-732, 2014.

[30] J.-Y. Qi, L. Wang, D.-S. Gu, L.-H. Guo, W. Zhu, and M.-Z. Zhang, "Protect effects of Danlou Tablet (fftff) against murine myocardial ischemia and reperfusion injury in vivo," Chinese Journal of Integrative Medicine, 2016. 
[31] Y. Ma, Y. Gai, J. Yan, J. Li, and Y. Zhang, "Puerarin attenuates anoxia/reoxygenation injury through enhancing Bcl-2 associated athanogene 3 expression, a modulator of apoptosis and autophagy," Medical Science Monitor, vol. 22, pp. 977-983, 2016.

[32] L. Tang, D. Liu, X. Yi et al., “The protective effects of puerarin in cardiomyocytes from anoxia/reoxygenation injury are mediated by PKC $\varepsilon$," Cell Biochemistry and Function, vol. 32, no. 4, pp. 378-386, 2014.

[33] R. Jaffe, T. Charron, G. Puley, A. Dick, and B. H. Strauss, "Microvascular obstruction and the no-reflow phenomenon after percutaneous coronary intervention," Circulation, vol. 117, no. 24, pp. 3152-3156, 2008.

[34] Z. Yang, T. Hong, and Y. M. Liu, "Protection of Danlou Tablet on hyperlipidemia and vascular endothelial injury in rats," World Journal of Integrated Traditional and Western Medicine, vol. 5, no. 6, pp. 491-494, 2010.

[35] M. Hong, "Effects of Danlou Tablet on myocardial infarction size and ventricular remodeling in rats," Chinese Journal of Experimental Traditional Medical Formulae, vol. 17, no. 10, pp. 208-211, 2011. 


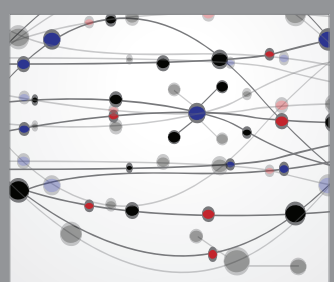

The Scientific World Journal
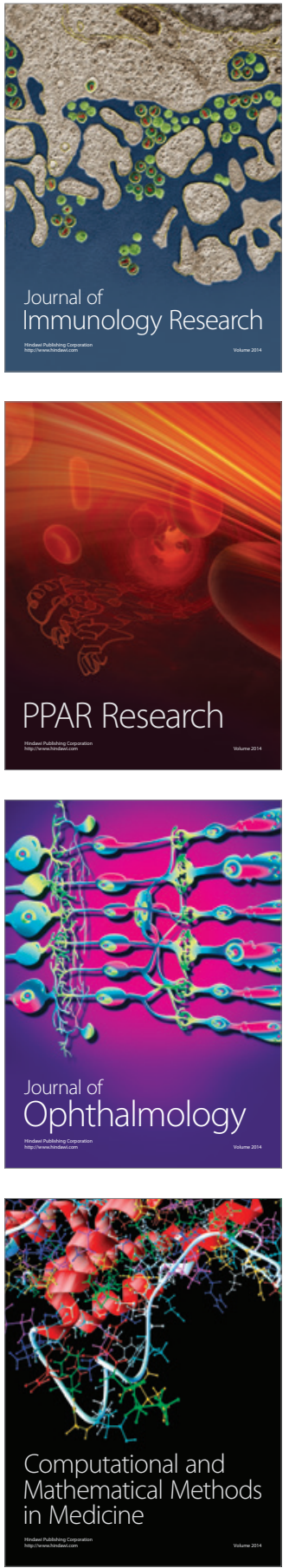

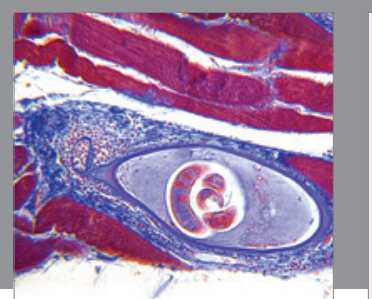

Gastroenterology Research and Practice

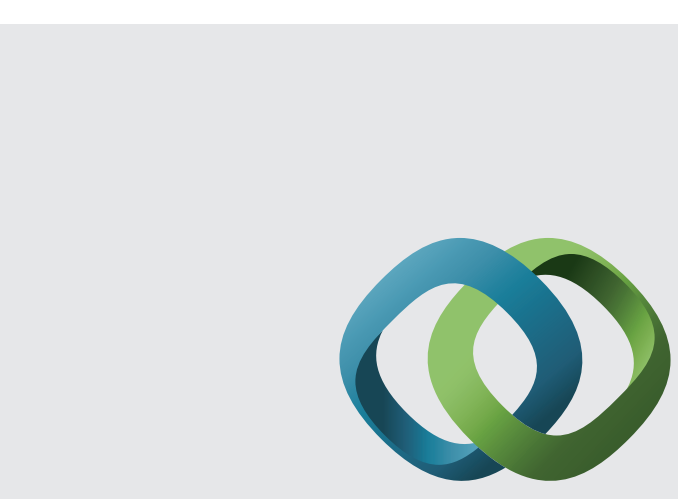

\section{Hindawi}

Submit your manuscripts at

http://www.hindawi.com
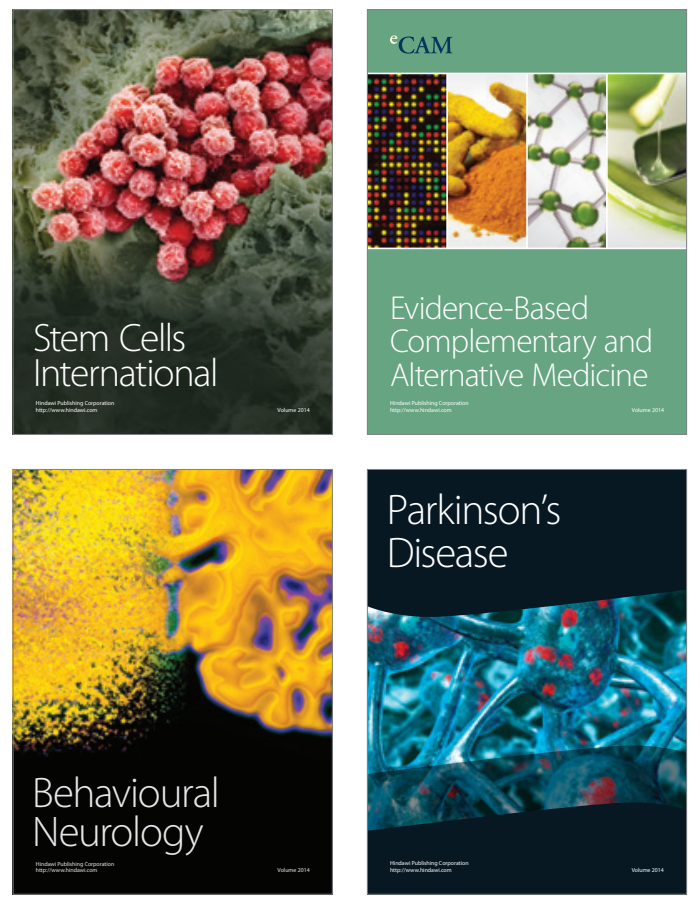
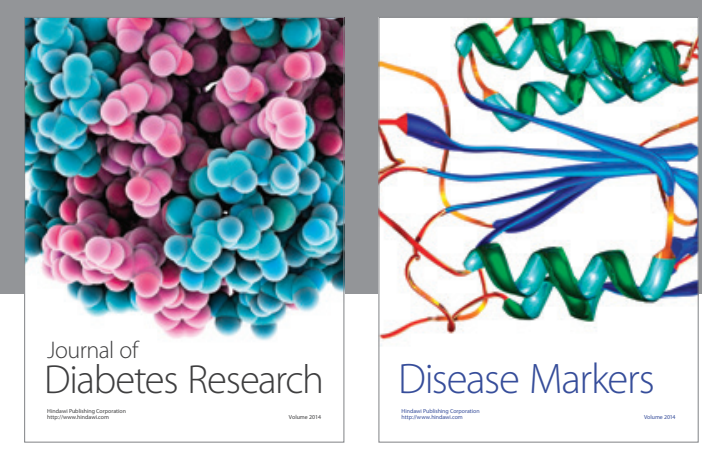

Disease Markers
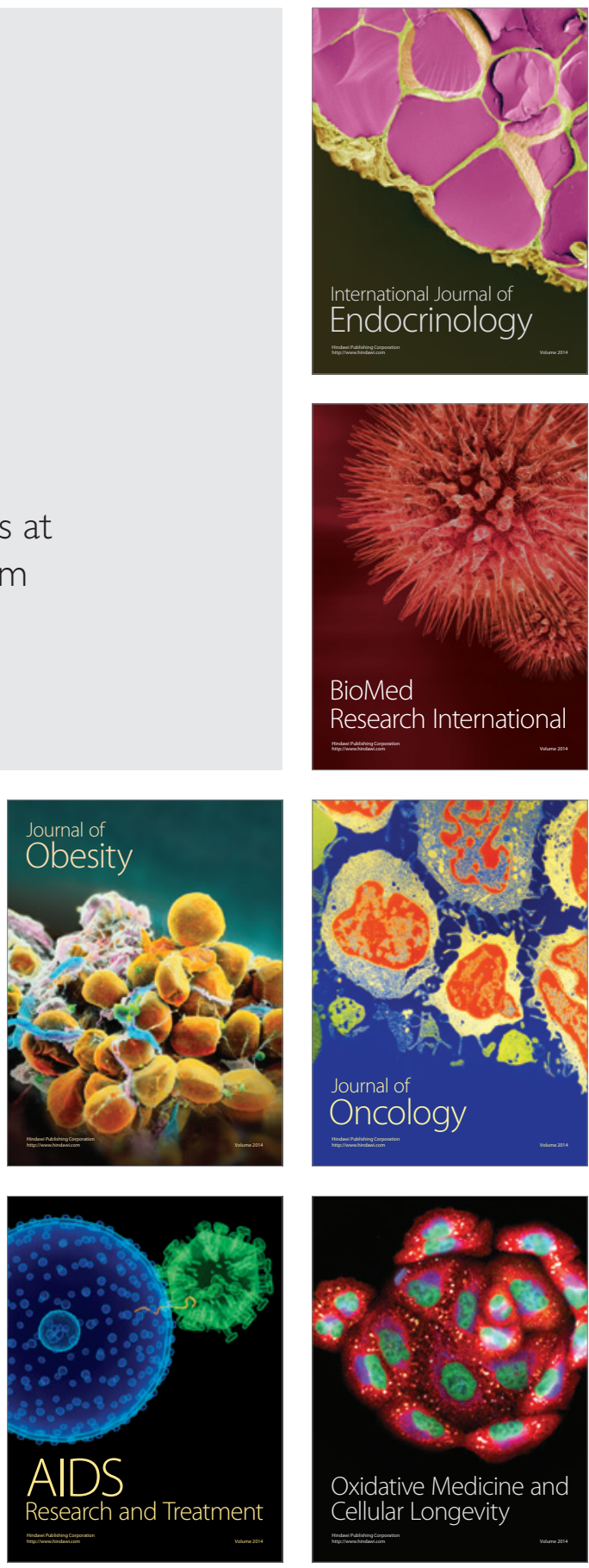